\title{
Wavelet analysis for polarization inhomogeneous laser images of blood plasma
}

\author{
O.G. Ushenko*, A.V. Dubolazov, V.O. Balanets'ka, A.V. Karachevtsev, M. Sydor \\ Optics and Spectroscopy Department \\ Chernivtsi National University \\ Chernivtsi, Ukraine, 58012
}

\begin{abstract}
The possibilities of the local wavelet-analysis of polarization-inhomogeneous laser image of human blood plasma were considered. The set of statistics, correlation and fractal parameters of the distributions of wavelet-coefficients that are characterize different scales of the polarization maps of polycrystalline networks of amino acids of blood plasma were defined. The criteria for the differentiation of the transformation of birefringence optical-anisotropic structures of blood plasma at different scales of their geometric dimensions were determined.

Keywords: birefringence, correlation functions, crystal, fractal, polarization, statistical moments, wavelet-analysis.
\end{abstract}

\section{INTRODUCTION}

Among many directions of optical diagnostics of organic phase-inhomogeneous objects, a new technique - laser polarimetry ${ }^{1,2}$ - has been formed within recent 10 years. It enables to obtain information about optical anisotropy of phase-inhomogeneous objects in the form of coordinate distributions of the biological tissues (BT) azimuths and ellipticities of their object field polarization ${ }^{1-5}$.

Specifically, the above mentioned model was used for finding and substantiating the interrelations between the ensemble of statistic moments of the $1^{\text {st }}$ to $4^{\text {th }}$ orders that characterize the orientation-phase structure (distribution of optical axes and phase shifts for directions of protein fibril networks) of birefringent BT architectonics and that of 2D distributions of azimuths and ellipticities of their laser images ${ }^{2}$. It was determined ${ }^{5}$ that the $3^{\text {rd }}$ and the $4^{\text {th }}$ statistic moments for coordinate distributions of ellipticities are the most sensitive to the change (caused by dystrophic and oncological processes) of optical anisotropy inherent to protein crystals. On this basis, the criteria for early diagnostics of muscle dystrophy, pre-cancer states of connective tissue, collagenosis, etc. were determined.

However, application of statistical analysis to coordinate distributions for azimuths and ellipticities of polarization in BT laser images does not enable to estimate local changes in the structure of optically anisotropic networks formed from protein crystals. On the other hand, in many cases the study of biological liquids (blood, urine, bile, synovial liquid, etc.) is more topical and accessible from the clinical viewpoint than the study of BT. Thereof, the task to develop new approaches to a local analysis of polarization-inhomogeneous images of biological liquids seems rather reasonable.

Our work is aimed at studying capabilities of the wavelet analysis in determination of statistical (statistical moments of the first to fourth orders) as well as fractal (fractal dimensions) parameters that characterize distributions of wavelet coefficients for images of blood plasma for diagnostics of oncological processes in a human organism.

\section{POLARIZATON MODELING OF PROPERTIES INHERENT TO NETWORKS OF BIOLOGICAL LIQUID CRYSTALS IN BLOOD PLASMA}

As a base for analyses of processes providing formation of polarization-inhomogeneous images of blood plasma, we use the optical model developed in ${ }^{3,4}$ :

- optical properties of blood plasma are determined as those of a two-component amorphous-crystalline structure;

- crystalline component is an architectonic net consisting of amino-acid liquid crystals;

- optically, the amino-acid liquid crystals possess the properties of uniaxial birefringent crystals.

*yuriyu@gmail.com

Tenth International Conference on Correlation Optics, edited by Oleg V. Angelsky, Proc. of SPIE

Vol. 8338, 83381H · @ 2011 SPIE · CCC code: 0277-786X/11/\$18 · doi: 10.1117/12.920169

Proc. of SPIE Vol. $833883381 \mathrm{H}-1$ 
Polarization properties of local optically coaxial crystalline amino acid can be described with the following Mueller operator $\{z\}_{u}$

$$
\{z\}_{u}=\left\|\begin{array}{cccc}
1 & 0 & 0 & 0 \\
0 & z_{22} & z_{23} & z_{24} \\
0 & z_{32} & z_{33} & z_{34} \\
0 & z_{42} & z_{43} & z_{44}
\end{array}\right\|,
$$

where

$$
z_{i k}(\rho, \delta)=\left\{\begin{array}{l}
z_{22}=\cos ^{2} 2 \rho+\sin ^{2} 2 \rho \cos \delta, \\
z_{23,32}=\cos 2 \rho \sin 2 \rho(1-\cos \delta), \\
z_{33}=\sin ^{2} 2 \rho+\cos ^{2} 2 \rho \cos \delta, \\
z_{34,43}= \pm \cos 2 \rho \sin \delta, \\
z_{24,42}= \pm \sin 2 \rho \sin \delta, \\
z_{44}=\cos \delta .
\end{array}\right.
$$

Here, $\rho$ is the direction of optical axis, $\delta=2 \pi / \lambda \Delta n d$ - phase shift introduced between the orthogonal components of the amplitude of laser wave with the length $\lambda$ passing through the liquid crystal with the linear size of its geometrical section $d$ and birefringence index $\Delta n$.

Mueller matrix $f_{i k}$ elements of liquid crystal network are determined by the following algorithm

$$
f_{i k}=\sum_{u=1}^{N}\left[z_{i k}(\rho, \delta)\right]_{u},
$$

where $N$ is a finite number of liquid crystals.

The classical definition of the Mueller matrix $\{F\}$ for biological objects consists not only in the fact that it describes optical properties of their optically anisotropic component, but also in the fact that such mathematical operator completely characterizes the processes of transformation of the Stokes vector $S$ by phase-inhomogeneous layers ${ }^{13}$

$$
S^{*}=\{F\} S_{0} .
$$

Here, $S_{0}, S^{*}$ are the Stokes vectors of illuminating and object beams.

For a more general state of elliptically polarized wave, the Stokes vector looks as follows

$$
S_{0}=\left(\begin{array}{l}
1 \\
\cos 2 \alpha_{0} \cos 2 \beta_{0} \\
\sin 2 \alpha_{0} \cos 2 \beta_{0} \\
\sin 2 \beta_{0}
\end{array}\right),
$$

where $\alpha_{0}, \beta_{0}$ are the azimuth and ellipticity of an electromagnetic wave.

Taking into account the expressions (2) to (5), the Stokes vector $S^{*}$ can be written in a complete form as 


$$
S^{*}=\left(\begin{array}{l}
1 \\
S_{2} \\
S_{3} \\
S_{4}
\end{array}\right)=\left(\begin{array}{l}
1 \\
f_{22} \cos 2 \alpha_{0} \cos 2 \beta_{0}+f_{23} \sin 2 \alpha_{0} \cos 2 \beta_{0}+f_{24} \sin 2 \beta_{0} \\
f_{32} \cos 2 \alpha_{0} \cos 2 \beta_{0}+f_{33} \sin 2 \alpha_{0} \cos 2 \beta_{0}+f_{34} \sin 2 \beta_{0} \\
f_{42} \cos 2 \alpha_{0} \cos 2 \beta_{0}+f_{43} \sin 2 \alpha_{0} \cos 2 \beta_{0}+f_{44} \sin 2 \beta_{0}
\end{array}\right)=\left(\begin{array}{l}
1 \\
\cos 2 \alpha \cos 2 \beta \\
\sin 2 \alpha \cos 2 \beta \\
\sin 2 \beta
\end{array}\right) .
$$

Being based on (6), we obtain expressions for determining the ellipticity $\beta$ of the object electromagnetic field polarization

$$
\beta=0.5 \arcsin \left(S_{4}\right) \equiv Q\left[f_{i k}(\rho, \delta), \alpha_{0}, \beta_{0}\right]
$$

It follows from the analysis of relation (7) that the state of polarization $(\beta)$ of each point $(r \equiv x, y)$ of the BT image is determined by corresponding local orientation-phase $(\rho, \delta)$ parameters of crystalline network.

In other words, on the terms of coordinate heterogeneity of distributions $\rho(r)$ and $\delta(r)$ in the plane of the BT layer a certain polarizationally inhomogeneous image is formed with distribution $\beta(r)$ called as polarization map (PM) ${ }^{5,23,2830}$.

\section{WAVELET APPROACH TO THE ANALYSIS OF DISTRIBUTIONS FOR ELLIPTICITY OF POLARIZATION OF LASER IMAGES INHERENT TO BLOOD PLASMA}

If a prototype function is taken as a specific wavelet function possessing a finite base both in coordinate and frequency spaces, then one can expand into series the one-dimensional distribution of ellipticity $\beta(x)$ for polarization

$$
\beta(x)=\sum_{a, b=-\infty}^{\infty} C_{a b} \Psi_{a b}(x),
$$

where $\Psi_{a b}(x)=\Psi(a x-b)$ is a base function formed from the function-prototype by shifting $\boldsymbol{b}$ and scaling $\boldsymbol{a}$, while the coefficients of this expansion are determined as follows

$$
C_{a b}=\int \beta(x) \Psi_{a b}(x) d x .
$$

The result of this wavelet transformation for the one-dimensional distribution of polarization parameters is a twodimensional array of the coefficients $W_{\beta}(a, b)$ that are defined by the following relation

$$
W(a, b)=\frac{1}{|a|^{1 / 2}} \int_{-\infty}^{+\infty} f(\beta) \Psi\left(\frac{x-b}{a}\right) d x .
$$

In our work, as a wavelet function we have used the so-called MHAT function, i.e. the second derivative of the Gauss function. It has been shown that MHAT wavelet possesses a narrow energy spectrum and two moments (zero and first) that are equal to zero. It satisfies the analysis of complex signals rather well. The mathematical expression for the MHAT wavelet is of the following form

$$
\Psi(x)=\frac{d^{2}}{d x^{2}} e^{-x^{2} / 2}=\left(1-x^{2}\right) e^{-x^{2} / 2} .
$$

To estimate $W_{\beta}(a, b=1,2, \ldots m)$ distributions for various scales $\boldsymbol{a}$ of the wavelet function $\Psi$, we calculated the set of their statistical moments of the first to fourth orders $M_{j=1 ; 2 ; 3 ; 4}{ }^{5}$

$$
\begin{array}{cl}
M_{1}=\frac{1}{m} \sum_{i=1}^{m}\left|W_{i}\right|, & M_{2}=\sqrt{\frac{1}{m} \sum_{i=1}^{m} W_{i}^{2}}, \\
M_{3}=\frac{1}{M_{2}^{3}} \frac{1}{m} \sum_{i=1}^{m} W_{i}^{3}, & M_{4}=\frac{1}{M_{2}^{4}} \frac{1}{m} \sum_{i=1}^{m} W_{i}^{4} .
\end{array}
$$

To calculate autocorrelation functions $W_{\beta}(a, b=1,2, \ldots m)$ related with wavelet coefficients for distributions of azimuths and ellipticity of laser image polarization, we used the following expression 


$$
\left.K(\Delta x)=\lim _{x \rightarrow 0} \frac{1}{N} \int_{0}^{x}[W(a ; x=1 \div m)] W(a ; x-\Delta x)\right] d x .
$$

Here, $(\Delta x)$ is the step of changing the coordinate $(b)$ for one-dimensional distributions of ellipticity $\beta$ of polarization. The fractal analysis of $W_{\beta}(a, b=1,2, \ldots m)$ distributions was performed using calculation of logarithmic dependences $\log J(W)-\log d^{-1}$ for power spectra $J(W)$

$$
J(W)=\int_{-\infty}^{+\infty} W_{a, b}(\beta) \cos 2 \pi v d v,
$$

where $v=d^{-1}$ are spatial frequencies determined by geometrical sizes $(d)$ of structural elements of the laser images for a biological layer.

The dependences $\log J(W)-\log \left(d^{-1}\right)$ are approximated using the least-squares method by the curves $\Phi(\eta)$, the straight parts of which are used to determine the slope angles $\eta$ and to calculate the value of fractal dimensionality $D$ via the relation

$$
D=3-\operatorname{tg} \eta
$$

Classification of $W_{\beta}(a, b=1,2, \ldots m)$ distributions is performed using the following criteria offered $\mathrm{in}^{5}$ :

- these distributions are fractal, on the condition that the slope angle has a constant value $\eta=$ const in the dependence $\Phi(\eta)$ within 2 or 3 decades in changing sizes $d$;

- the distributions are multi-fractal, on the condition of availability of several constant values for slope angles in $\Phi(\eta)$;

- the distributions are random, if there is no stable slope angle in $\Phi(\eta)$ over the whole range of changing sizes $d$.

\section{OPTICAL SCHEME OF THE POLARIMETER AND TECHNIQUE OF POLARIMETRIC INVESTIGATIONS}

Figure 1 shows the traditional optical scheme of a polarimeter for measuring the set of coordinate distributions for azimuths and ellipticity of polarization of laser images inherent to human blood plasma.

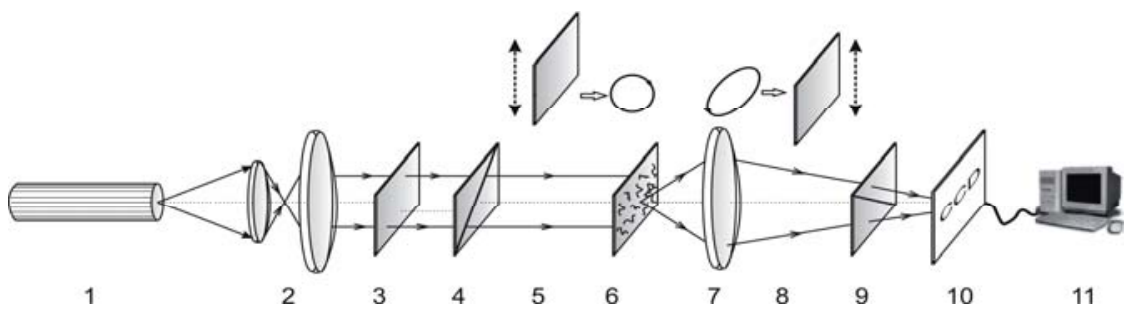

Figure 1. Optical scheme of the polarimeter. 1 - He-Ne laser; 2 - collimator; 3 - stationary quarter-wave plate; 5, 8 - movable quarterwave plates; 4, 9 - polarizer and analyzer, respectively; 6 - object under investigation; 7 - micro-objective; 10 - CCD camera; 11 personal computer.

Illumination was made with a collimated beam (radius $r=10 \mathrm{~mm}$ ) of He-Ne laser $1(\lambda=0.6328 \mu \mathrm{m})$. Using the polarization illuminator (quarter-wave plates 3,5 and polarizer 4) we formed respective states for polarization of illuminating beam: $1-0^{0} ; 2-90^{0} ; 3-45^{0} ; 4-\otimes$ (right circulation).

The image of blood plasma layer was formed within the light-sensitive area ( 800 pix $\times 600$ pix $)$ of CCD camera 10 by using the micro-objective 7.

For each separate pixel, we determined four parameters of the Stokes vector 


$$
\begin{aligned}
& S_{1}=I_{0}+I_{90} ; \\
& S_{2}=I_{0}-I_{90} ; \\
& S_{3}=I_{45}-I_{135} ; \\
& S_{4}=I_{\otimes}-I_{\oplus} .
\end{aligned}
$$

Here, $I_{0}, I_{90}, I_{45}, I_{135}$ are the intensities of linearly (with the azimuths $0^{0}, 90^{0}, 45^{0}, 135^{\circ}$ ) as well as left- $I_{\oplus}$ and right$I_{\otimes}$ circularly polarized radiation transmitted by the system of the quarter-wave 8 - polarizer 9 .

The values of azimuth and ellipticity of polarization were calculated using the following algorithms

$\beta(m \times n)=0,5 \arcsin \left[S_{4}(m \times n) / S_{1}(m \times n)\right]$.
The coordinate sets of values $\beta\left(\begin{array}{ccc}r_{11} & \ldots & r_{1 n} \\ \ldots & \ldots & \ldots \\ r_{m 1} & \ldots & r_{m n}\end{array}\right)$ we shall name as polarization map.

\section{BRIEF CHARACTERISTIC OF THE OBJECTS UNDER INVESTIGATION}

The main optically anisotropic elements of blood plasma are albumin and globulin that form networks of liquid crystals in the process of crystallization. The structure of these networks is superposition of albumin crystals with spatially ordered directions of optical axes and spatially disordered globulin crystals.

The above model assumptions can be qualitatively illustrated by the results of comparative investigation of structures in laser images of blood plasma taken from healthy and sick patients (figure 2).

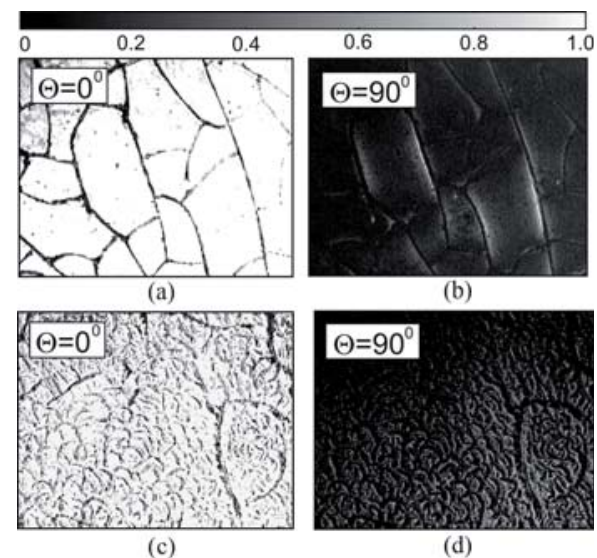

Figure 2. Polarization images of liquid-crystalline optically anisotropic network inside blood plasma of healthy (a), (b) and sick (c), (d) patients.

As seen from these images, the blood plasma of a healthy patient is characterized with domination of a large-scale network consisting of albumin crystals with ordered directions of their optical axes. In the laser images of the blood plasma taken from a patient with cervical carcinoma, one can observe domination of small-scale disordered networks consisting of albumin crystals. 


\section{POLARIZATION MAPS FOR LASER IMAGES OF HUMAN BLOOD PLASMA}

Figure 3 illustrate coordinate distributions of ellipticity $\beta(m \times n)$ (figure $3 \mathrm{a}$ ) of polarization; histograms of distributions for their values $h(\beta)$ (figure 3b); autocorrelation functions $C(\Delta x)$ for $\beta(m \times n)$ distributions (figure 3c); as well as $\operatorname{logarithmic~dependences~} \log J(\beta)-\log d^{-1}$ (figure 3d) for polarization maps of blood plasma taken from healthy (top ) and sick (bottom) patients.

The analysis of coordinate distributions for ellipticity $\beta(m \times n)$ (figure 3a) has shown that they contain two components:

- large-scale (100 to $300 \mu \mathrm{m})$ parts with homogeneous polarization (images of the optically isotropic component in human blood plasma) that coincides with that of laser beam $\alpha^{*}=\alpha_{0}=0^{0}$;

- polarization-inhomogeneous parts $\beta(\Delta x, \Delta y)=$ const - laser images of elements (2 to $50 \mu \mathrm{m})$ of albumin and globulin crystalline network.

Quantitatively this structure of polarization maps for blood plasma of both types can be illustrated with histograms $h(\beta)$ that are dependences symmetrical relatively to the main extrema at $\alpha=0^{0} ; \beta=0^{0}$.

Summarized in Table 1 are the values and ranges for statistical moments of the first to fourth orders that characterize distributions $\beta(m \times n)$ within the limits of two groups of healthy $(q=21)$ and oncologically sick $(q=19)$ patients.

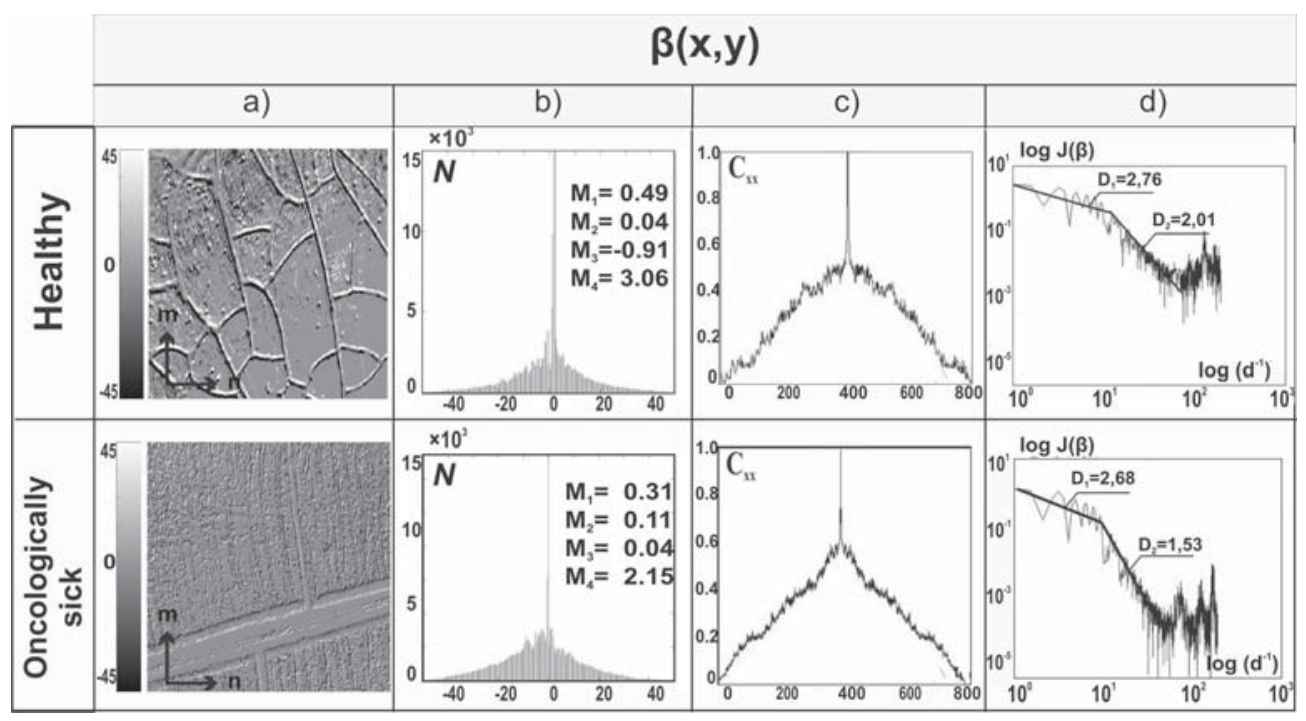

Figure 3. Polarization maps for ellipticity $\beta(m \times n)(\mathrm{a}, \mathrm{e})$, their statistical $(\mathrm{b}, \mathrm{f})$, correlation $(\mathrm{c}, \mathrm{g})$, and fractal (d,) parameters of blood plasma taken from healthy (left column) and oncologically sick (right column) patients.

Table 1. Statistical moments of the first to fourth orders for distributions of polarization parameters in laser images of human blood plasma in different physiological states of patients.

\begin{tabular}{|c|c|c|}
\hline$M_{j}(\beta)$ & $\begin{array}{c}\text { Norm } \\
(q=21)\end{array}$ & $\begin{array}{c}\text { Oncology } \\
(\mathrm{q}=19)\end{array}$ \\
\hline$M_{1}(\beta)$ & $0.49 \pm 0.055$ & $0.31 \pm 0.058$ \\
\hline$M_{2}(\beta)$ & $0.04 \pm 0.007$ & $0.11 \pm 0.006$ \\
\hline$M_{3}(\beta)$ & $0.91 \pm 0.09$ & $0.04 \pm 0.094$ \\
\hline$M_{4}(\beta)$ & $3.16 \pm 0.33$ & $2.15 \pm 0.31$ \\
\hline
\end{tabular}

Our comparative analysis of the data obtained did not reveal sufficiently reliable criteria (within the framework of statistical approach) for differentiation of coordinate structure in polarization maps for blood plasma of both types. The 
values and ranges for changing the whole set of statistical moments $M_{j=1,2,3,4}$ related to distributions of $\beta$ ellipticity of polarization are superimposed.

Correlation and fractal analyses of polarization maps describing blood plasma taken from healthy and oncologically sick patients revealed a fractal structure inherent to coordinate distributions of ellipticity $\beta(m \times n)$ of polarization within the range of mean $(50$ to $200 \mu \mathrm{m})$ and large $(200$ to $2000 \mu \mathrm{m})$ geometrical sizes of amino acid biological crystals. The approximating curves $\Phi(\eta)$ for dependences $\log J(\beta)-\log d^{-1}$ (fugure 3d,h) are characterized with stable values of slope angles $\eta(\beta) \rightarrow\left\{\begin{array}{l}D_{\beta(1)}=2,76 ; D_{\beta(1)}^{*}=2,68 ; \\ D_{\beta(2)}=2,01 ; D_{\beta(2)}^{*}=1,53\end{array}\right.$.

In the field of small geometric sizes ( 2 to $50 \mu \mathrm{m})$ of the polycrystalline network inherent to amino acids in human blood plasma for the patient with cervical carcinoma, the values of fractal dimensionalities $D_{\beta}^{*}$ for distributions of polarization parameters become indefinite. $\Phi_{\beta}(\eta)$ dependences within this range of sizes inherent to amino acid crystals are curve without any definite slope angle $-\eta(2 . .50 \mu m) \neq$ const .

In our opinion, this "destruction" of self-similarity for the distributions $\beta(m \times n)$ in polarization maps inherent to blood plasma of oncologically sick patient is related with formation of a network of small-scale globulin crystals (Fig. 2c,d). By other words, to determine a set of objective criteria for differentiation of polycrystalline networks of both types, one needs a more detailed analysis of polarization distributions just for these scales of geometrical sizes of biological crystals.

\section{WAVELET ANALYSIS OF POLARIZATION DISTRIBUTIONS OF LASER IMAGES FOR POLYCRYSTALLINE NETWORKS IN BLOOD PLASMA}

The locally-scaled analysis of coordinate distributions $\beta(m \times n)$ for laser images of blood plasma is provided using linear $k_{1}, \ldots, k_{m}, k=1 \div n$ scanning by the MHAT wavelet with the step $b=1$ pix and window width $1 \mu \mathrm{m} \leq a_{\min } \leq 70 \mu \mathrm{m}$. The result of this scanning can be represented (see relation (11)) as a two-dimensional set of wavelet coefficients $W_{a, b}=\left(\begin{array}{ccc}W\left(a_{\min }, b_{1}\right) & \ldots & W\left(a_{\min }, b=m\right. \\ \ldots & \ldots & \ldots \\ W\left(a_{\max }, b_{1}\right. & \ldots & W\left(a_{\max }, b=m\right.\end{array}\right)$ for each $k-$ th line of pixels (figure $\left.4 \mathrm{a}, \mathrm{e}\right)$ in the lightsensitive area of CCD 10 (figure 1).

Thus, the obtained set of wavelet coefficients $W\left(a_{\min } ; b=k_{1} \div k_{m}\right)$ should be averaged using the following algorithm

$$
\bar{W}_{a, b}=\left(\begin{array}{ccc}
\bar{W}\left(a_{\min }, b_{1}\right)=\frac{\sum_{j=1}^{m} W_{j}\left(a_{\min }, b_{1}\right)}{m} & \ldots & \bar{W}\left(a_{\min }, b=m\right)=\frac{\sum_{j=1}^{m} W_{j}\left(a_{\min }, b=m\right)}{m} \\
\bar{W}\left(a_{\max }, b_{1}\right)=\frac{\sum_{j=1}^{m} W_{j}\left(a_{\max }, b_{1}\right)}{m} & \ldots & \\
m & \ldots & \bar{W}\left(a_{\max }, b=m\right)=\frac{\sum_{j=1}^{m} W_{j}\left(a_{\max }, b=m\right)}{m}
\end{array}\right) .
$$

The algorithm (18) is an analog of two-dimensional wavelet transformation that characterizes coordinate distributions for ellipticity $\beta(m \times n)$ (Fig. 3a,e) of polarization observed in laser images within the range of small scales $1 \mu \mathrm{m} \leq a_{\min } \leq 70 \mu \mathrm{m}$ in polycrystalline structures of blood plasma.

Shown in figures 5 and 6 are the results of experimental investigations of statistical (statistical moments of the 1-st to 4th orders $M_{j=1,2,3,4}\left(W_{a, b}\right)$, correlation (autocorrelation functions $K\left(W_{a, b}\right)$ ) and fractal (logarithmic dependences of the power spectra $\left.\log J\left(W_{a, b}\right)-\log d^{-1}\right)$ parameters that characterize the distributions $W\left(a_{\min } ; b=k 1 \div k m\right)$ for two scales $a_{\min }=2 ; a_{\min }=30$ of the MHAT wavelet for polarization maps $\beta(m \times n)$ describing blood plasma of a healthy (left column) and sick (right column) patients. 
As seen from the data obtained, the distributions for wavelet coefficients $\bar{W}\left[\left(a_{\min }=2 ; 30\right) ;(b=k 1 \div k m)\right]$ of polarization maps for the polycrystalline network of amino acids from healthy patient's blood plasma, which is ordered along directions of optical axis (figure 2a), are individual for each scale $a_{\min }$ of the MHAT wavelet.
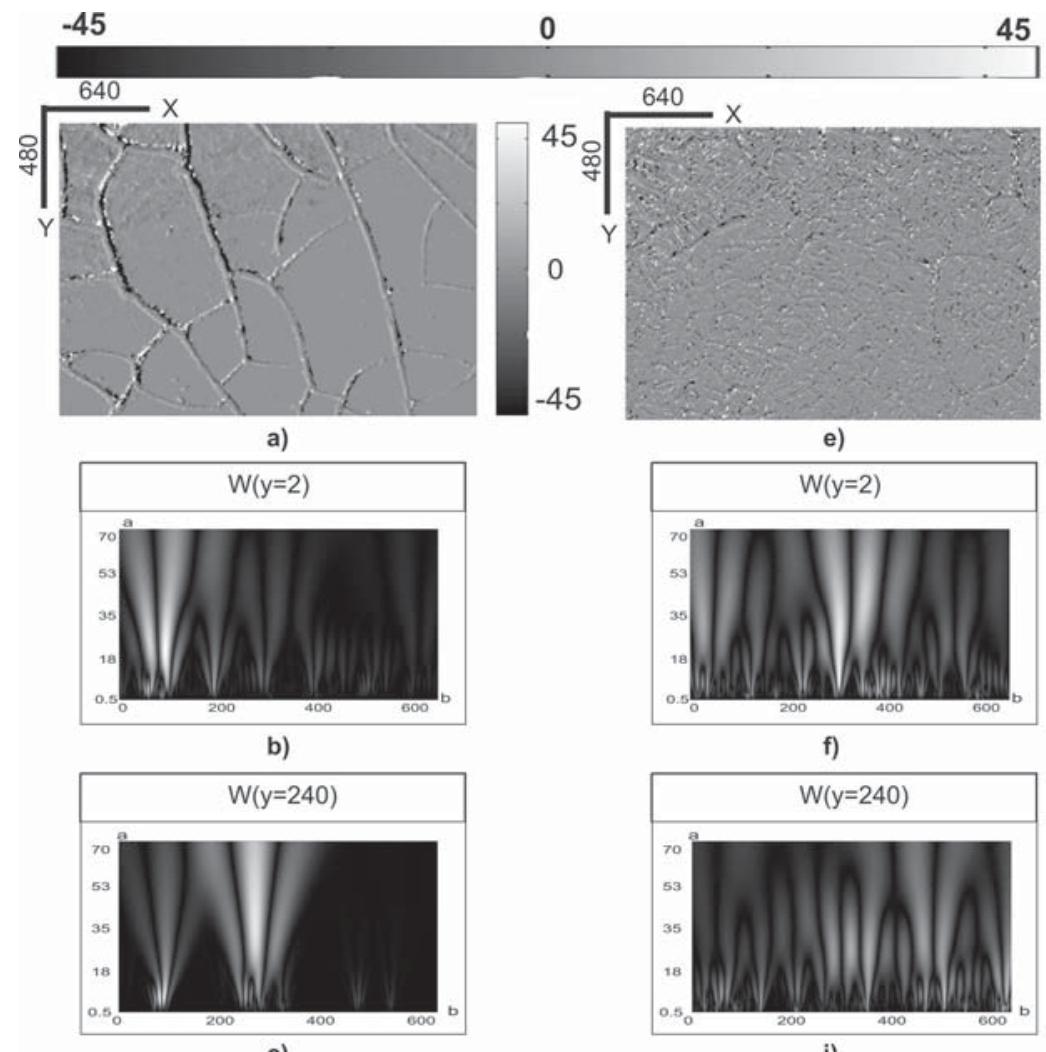

c)

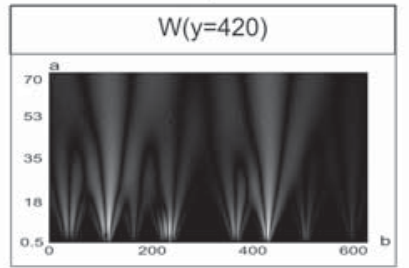

d)

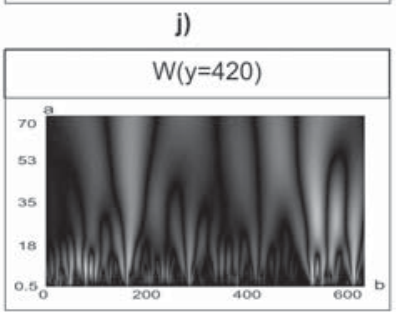

h)

Figure 4. Distributions of wavelet coefficients $W\left(a_{\min } ; b=k_{1} \div k_{m}\right)$ of the polarization map for ellipticity $\beta(m \times n)$ of polarization observed in blood plasma of a healthy (left column) and sick (right column) patient's blood plasma for various lines $k=2,240,420$ of CCD-camera.

Our statistical analysis (figures 5 and 6) of the distributions $\bar{W}_{\left[\left(a_{\min }=2 ; 30\right) ;(b=k 1 \div k m)\right]}(\beta)$ revealed different dynamics for changing the values $M_{j=1 ; 2 ; 3 ; 4}$ (fragments a,d) with increasing the scale $a_{\min }$ of the MHAT wavelet. The ranges of changes in statistical moments of the 1-st to 4-th orders lie within the limits of $M_{3}=2 \div 6$ and $M_{4}=3 \div 11$ times, respectively. The found tendency is indicative of transformation observed for distributions of wavelet coefficients from practically random $\left(M_{1 ; 2}>>M_{3 ; 4} \rightarrow 0\right)$ up to the stochastic ones $\left(M_{3 ; 4}>>M_{1 ; 2}\right)$. 

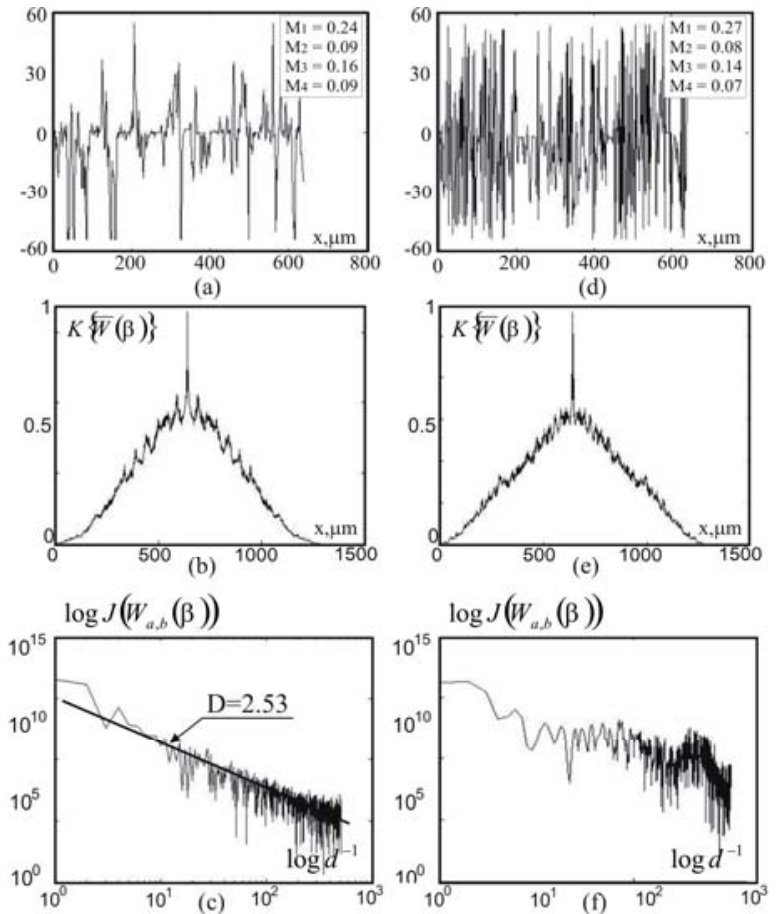

Figure 5. Statistical (left column), correlation (central column) and fractal (right column) parameters of distributions inherent to wavelet coefficients $\bar{W}\left[\left(a_{\min }=2\right) ;(b=k 1 \div \mathrm{km})\right]$ describing the polarization map for $\beta(m \times n)$ of a healthy (left column) and sick (right column) patient's blood plasma.
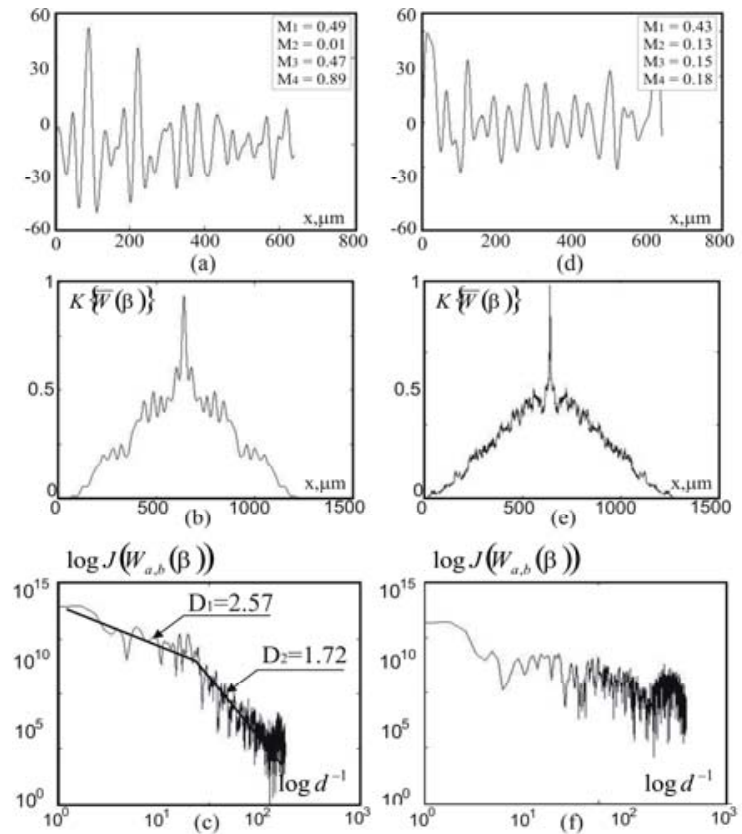

Figure 6. Statistical (left column), correlation (central column) and fractal (right column) parameters of distributions inherent to wavelet coefficients $\bar{W}\left[\left(a_{\min }=30\right) ;(b=k 1 \div k m)\right]$ describing the polarization map for $\beta(m \times n)$ of a healthy (left column) and sick (right column) patient's blood plasma. 
This fact is also confirmed by the dependences (Figures 5 and 6 , fragments a,d) of the autocorrelation functions $K\left\{\bar{W}_{\left[\left(a_{\min }=2 ; 10 ; 30\right) ;(b=k 1 \div \mathrm{km})\right]}(\beta)\right\}$ for the distributions of wavelet coefficients, as they are superposition of two components, namely: statistical that drops monotonically, and the oscillating one that is caused by periodical coordinate changes in these distributions.

The revealed features of statistical and coordinate structures in distributions of wavelet coefficients for polarization maps describing healthy patient's blood plasma are related, in our opinion, with a different degree of self-similarity in distributions of optical axis directions $\rho$ and phase shifts $\delta$ in polycrystalline structures at different scales of analysis $a_{\min }$ of the MHAT wavelet. So, for small scales $\left(a_{\min }=2 \mu \mathrm{m}\right)$, a dominant contribution to formation of the coordinate distributions $\beta(m \times n)$ is caused by chaotically oriented globulin crystals. Therefore, just the random component dominates in the respective distributions for wavelet coefficients $\bar{W}_{\left[\left(a_{\min }=2\right) ;(b=k 1 \div k m)\right]}(\beta)$.

When the scale grows $\left(a_{\min }=30\right)$, also growing is the contribution to formation of distributions for polarization parameters of the set oriented along directions of optical axes of albumin crystals. From the statistical viewpoint, this process should be observed in the growth of statistical moments of the 3-rd and 4-th orders that characterize the distributions $\bar{W}_{\left[\left(a_{\min }=30\right) ;(b=k 1 \div k m)\right]}(\beta)$, as well as in formation of oscillations of autocorrelation dependences $K\{\bar{W}(\beta)\}$.

Besides, some stable slope $\eta$ of approximating curves $\Phi(\eta)$ for $a_{\min }=2$ in the logarithmic dependences $\log J\left(W_{a, b}(\beta)\right)-\log d^{-1}$ is absent. The growth in the scale $\left(a_{\min }=30\right)$ of the MHAT wavelet can be observed in transformation of the random curves $\Phi(\eta)$ into polygonal lines (figures 5 and 6 , fragments $\mathrm{c}, \mathrm{f}$ ). In other words, the random distributions $\bar{W}_{\left[\left(a_{\min }=2\right) ;(b=k 1 \div k m)\right]}(\beta)$ are transformed into the multi-fractal ones.

Our analysis of the data obtained for statistical, correlation and fractal parameters that characterize sets of wavelet coefficients for various scales of MHAT functions for distributions of ellipticity $\beta(m \times n)$ of laser images of the albumin-globulin polycrystalline network in blood plasma of a patient with cervical carcinoma enabled us to find:

1) Weak changes (within 10 to $15 \%$ ) of the values of statistical moments $M_{j=1 ; 2 ; 3 ; 4}$ that characterize the distributions $\bar{W}_{\left[\left(a_{\min }=2\right) ;(b=k 1 \div k m)\right]}(\beta)$ on the scales $a_{\min }=2 \mu m$ of the MHAT wavelet as compared with analogous statistical parameters determined for polarization maps of healthy patient's blood plasma.

2) An essential decrease of statistical moments of the 3-rd (2.7 to 3.5 times) and 4-th (3.4 to 5.7 times) orders for the distributions $\bar{W}_{\left[\left(a_{\min }=2 ; 10 ; 30\right) ;(b=k 1 \div k m)\right]}(\beta)$ determined on larger scales $\left(a_{\min }=30\right)$ of the MHAT wavelet.

3) A faster drop in the autocorrelation dependences $K\{\bar{W}(\beta)\}$ as well as decrease in their fluctuation amplitudes.

4) The absence of any stable slope of the approximating curves $\Phi(\eta)$ for the logarithmic dependences $\log J\left(W_{a, b}(\beta)\right)-\log d^{-1}$ determined on all the scales of the MHAT wavelet.

The above mentioned differences between statistical moments, autocorrelation functions and logarithmic dependences that characterize the distributions $\bar{W}_{\left[\left(a_{\min }=30\right) ;(b=k 1 \div k m)\right]}(\beta)$ can be related with growth of the albumin concentration in blood plasma of a patient with the oncological process.

This biochemical process results in growth of the birefringence coefficient for partial albumin crystals disordered as to directions of their optical axes. And this transformation of the polycrystalline structure begins from small sizes $(d=1 \mu m \div 50 \mu m)$ of structural elements in the polycrystalline network.

From the viewpoint of polarization, these processes become apparent via formation of random distributions for ellipticity $\beta(m \times n)$ (figure 3e) in respective blood plasma laser images obtained in the case of an oncologically sick patient. It results in decrease of the values inherent to statistical moments of the 3-rd and 4-th orders that characterize the distributions $\bar{W}_{\left[\left(a_{\min }=2 ; 30\right) ;(b=k 1 \div k m)\right]}(\beta)$ on all the scales $a_{\min }$ of the MHAT wavelet.

Due to the same reason, autocorrelation functions experience faster drop of their intrinsic values, while the approximating curves $\Phi(\eta)$ for the logarithmic dependences $\log J(W(\beta))-\log d^{-1}$ are characterized with the absence of a stable slope angle.

Possibilities to diagnose pathological processes in a human organism by using the wavelet analysis of polarization maps for ellipticity of laser images describing blood plasma have been illustrated in Table 2, where the values of statistical 
moments that characterize distributions on three scales $a_{\min }$ of the MHAT wavelet for two groups of healthy (21 samples) and sick (19 samples) patients are summarized.

Table 2. Statistical moments of the 1-st to 4-th orders for distributions of wavelet coefficients related to polarization maps for ellipticity $\beta(m \times n)$ of laser images describing blood plasma of a healthy patient and oncologically sick.

\begin{tabular}{|c|c|c|c|}
\hline \multirow{3}{*}{$a_{\min }=2$} & $M_{1}$ & $0.24 \pm 0.028$ & $0.27 \pm 0.032$ \\
\cline { 2 - 4 } & $M_{2}$ & $0.09 \pm 0.01$ & $0.08 \pm 0.009$ \\
\cline { 2 - 4 } & $M_{3}$ & $0.16 \pm 0.024$ & $0.14 \pm 0.018$ \\
\cline { 2 - 4 } & $M_{4}$ & $0.09 \pm 0.009$ & $0.07 \pm 0.0085$ \\
\hline \multirow{5}{*}{$a_{\min }=30$} & $M_{1}$ & $0.49 \pm 0.053$ & $0.43 \pm 0.051$ \\
\cline { 2 - 4 } & $M_{2}$ & $0.1 \pm 0.017$ & $0.13 \pm 0.017$ \\
\cline { 2 - 4 } & $M_{3}$ & $0.47 \pm 0.058$ & $0.15 \pm 0.019$ \\
\cline { 2 - 4 } & $M_{4}$ & $0.89 \pm 0.092$ & $0.16 \pm 0.018$ \\
\hline
\end{tabular}

\section{CONCLUSION}

Thus, we have demonstrated diagnostic efficiency of the wavelet analysis applied to coordinate distributions for ellipticity of polarization in laser images inherent to amino acid polycrystalline networks in blood plasma of patients with oncological changes in woman's genital organs.

\section{REFERENCES}

[1] de Boer, J.F. and Milner, T.E., "Review of polarization sensitive optical coherence tomography and Stokes vector determination," J. Biomed. Opt. 7, 359-371 (2002).

[2] de Boer, J.F., Milner T.E. and Nelson J.S., [Trends in Optics and Photonics (TOPS): Advances in Optical Imaging and Photon Migration], Washington, OSA (1998).

[3] Everett, M.J., Shoenenberger, K., Colston, B.W. and da Silva, L.B., "Birefringence characterization of biological tissue by use of optical coherence tomography," Opt. Lett. 23, 228-230 (1998).

[4] Shuliang, J., Wurong, Yu., Stoica, G. and Lihong, V., "Optical fiber based Mueller optical coherence tomography," Opt. Lett. 28, 1206-1208 (2003).

[5] Angelsky, O.V., Maksimyak, P.P., Hanson, S.G., Ryukhin, V.V. "New Feasibilities for Characterizing Rough Surfaces by Optical-Correlation Techniques," Appl. Opt. 40, 5693-5707 (2001). 